\title{
Emotion and time perception: effects of film-induced mood
}

\author{
Sylvie Droit-Volet ${ }^{1 *}$, Sophie L. Fayolle ${ }^{1}$ and Sandrine Gil ${ }^{2}$ \\ 1 Laboratoire de Psychologie Sociale et Cognitive, CNRS, UMR 6024, Université Blaise Pascal, Clermont-Ferrand, France \\ ${ }^{2}$ Centre de Recherche sur la Cognition et l'Apprentissage, CNRS, UMR 6234, University of Poitiers, Poitiers, France
}

Edited by:

Valerie Doyere, CNRS, France

Reviewed by:

Ullrich Wagner, Charité - University

Medicine Berlin, Germany

Jason Tipples, University of Hull, UK

${ }^{*}$ Correspondence:

Sylvie Droit-Volet, Laboratoire de Psychologie Sociale et Cognitive,

CNRS, UMR 6024, Université Blaise

Pascal, 34 Avenue Carnot, 63037

Clermont-Ferrand, France.

e-mail:sylvie.droit-volet@

univ-bpclermont.fr
Previous research into emotion and time perception has been designed to study the time perception of emotional events themselves (e.g., facial expression). Our aim was to investigate the effect of emotions per se on the subsequent time judgment of a neutral, non-affective event. In the present study, the participants were presented with films inducing a specific mood and were subsequently given a temporal bisection task. More precisely, the participants were given two temporal bisection tasks, one before and the other after viewing the emotional film. Three emotional films were tested: one eliciting fear, another sadness, and a neutral control film. In addition, the direct mood experience was assessed using the Brief Mood Introspective Scale that was administered to the participants at the beginning and the end of the session. The results showed that the perception of time did not change after viewing either the neutral control films or the sad films although the participants reported being sadder and less aroused after than before watching the sad film clips. In contrast, the stimulus durations were judged longer after than before viewing the frightening films that were judged to increase the emotion of fear and arousal level. In combination with findings from previous studies, our data suggest that the selective lengthening effect after watching frightening films was mediated by an effect of arousal on the speed of the internal clock system.

Keywords: time perception, timing, emotion, mood, fear, sadness

\section{INTRODUCTION}

In everyday life, the experience of a mood changes our relationship with time. When we are sad and depressed we have the feeling that the flow of time slows down. Every hour seems like an eternity, as if time had stopped. In contrast, the feeling of stress seems to accelerate the flow of time. One hour seems nothing. Although these mood-related fluctuations in our experience of time have often been described, they have rarely been experimentally studied. Finally, we do not know whether these explicit judgments concerning the passage of time correspond to a reality experienced in our bodies or brains.

It may seem strange to speak about the reality of time since humans are not equipped with any specific sensory receptor enabling them to capture temporal information (for recent reviews, see Coull et al., 2011; Van Wassenhove et al., 2011). Nevertheless, the brain is inherently capable of processing time. Whatever the model of the temporal neural machinery (e.g., oscillators, climbing neurons, pulses), the subjective duration of events is thought to be based on cerebral units accumulated in time (e.g., Durstewitz, 2004; Matell and Meck, 2004; Karmarkar and Buonomano, 2007). Researchers have therefore posited the existence of an internal clock-like system that provides the raw material for the representation of time. According to the analogical internal clock models (Treisman, 1963; Gibbon et al., 1984), this clock consists of a pacemaker and an accumulator. At the beginning of the stimulus to be timed, a switch controlled by attention closes, and the pulses emitted by the pacemaker flow into the accumulator. The subjective duration thus depends on the number of pulses accumulated. When more pulses are accumulated, time is judged to be longer. Two main mechanisms have therefore been suggested to account for fluctuations in the passage of time: (1) an attention-based mechanism and (2) an arousal-based mechanism (Droit-Volet and Meck, 2007; Droit-Volet and Gil, 2009). In the case of the first mechanism, when attention is distracted away from the processing of time, the switch connecting the pacemaker to the accumulator opens, and some pulses are lost. The duration is therefore judged relatively shorter because fewer pulses have been accumulated. A shortening effect is therefore observed as the amount of attention devoted to time decreases. In the case of the second mechanism, a lengthening rather than a shortening effect occurs. When the level of arousal increases, the pacemaker is thought to speed up in line with the physiological activation of the organism. More pulses are emitted and the duration is judged longer. The mathematics underpinning the internal clock models predicts that the attention-based mechanism would result in an additive effect, with an attentional effect on switch closure latency that remains constant whatever the length of the stimulus duration. In contrast, the arousal-based speeding-up of the internal clock would result in a multiplicative effect accompanied by a lengthening effect that is relatively greater for long than for shorter stimulus durations (PentonVoak et al., 1996; Penney et al., 2000; Droit-Volet and Wearden, 2002). This has been verified in experiments which have used at least two different duration ranges (a short and a longer one) and manipulated the level of dopamine in the brain through the administration of psychostimulant drugs (methamphetamine, 
cocaine, caffeine; e.g., Maricq et al., 1981; Meck, 1983; Rammsayer, 1989, 2009).

As of some 10 years ago, researchers started to systematically investigate the mechanisms involved in the time distortions that are seen to occur in the presence of emotions. Unlike the pioneering psychologists who invented original situations without, however, perfectly controlling the emotional environment (e.g., Falk and Bindra, 1954; Langer et al., 1961; Thayer and Schiff, 1975), these researchers have used material taken from tested, validated databases of normative stimuli to elicit emotions in the laboratory (Coan and Allen, 2007). Studies of the perception of time have used images from the well-known International Affective Picture System (IAPS; Angrilli et al., 1997; Lang et al., 2008; Grommet et al., 2010). Pouthas and her collaborators also used sounds from the International Affective Digital Sounds (IADS; Bradley and Lang, 1999; Noulhiane et al., 2007; Mella et al., 2011). In our laboratory, we have mainly used emotional facial expressions (Droit-Volet et al., 2004; Effron et al., 2006; Gil et al., 2007; Gil and Droit-Volet, 2011a,b), which have since been used by other researchers (Tipples, 2008, 2011; Bar-Haim et al., 2009; Doi and Shinohara, 2009; Lee et al., 2011). These studies using different emotional stimuli have systematically shown that the presentation duration of negative high-arousing emotional stimuli was judged longer than that of neutral control stimuli. They thus logically explained this lengthening effect in terms of these emotional stimuli that physiologically activated the central nervous system, and accelerated the mechanisms which underpin the internal clock.

However, these different studies have investigated the judgment of the presentation duration of emotional stimuli rather than the effect of emotional states (mood) per se on the perception of time, i.e., on the perception of the duration of a neutral, non-affective stimulus. In the case of the processing of emotional stimuli, the non-temporal characteristics of the stimuli might interfere with the processing of time. As far as emotional sounds are concerned, the parameters of sounds (melodic contour, tempo, loudness, etc.) have been shown to affect the perception of time irrespective of their emotional dimension. For example, when listening to a piece of music, a feeling of happiness is induced when the music is played at a fast tempo in a major key compared to the feeling a sadness that results from hearing the music played at a slow tempo in a minor key (Peretz et al., 1998). Tempo is thus a critical factor influencing the experience of emotion. More specifically, when tempo is controlled, no effect of emotional valence on the perception of time is observed (Droit-Volet et al., 2010a). It is therefore difficult, on the basis of the temporal judgments, to distinguish between what is produced by the elicited emotion and the non-temporal characteristics of sound since the two are closely interlinked. This methodological problem is reduced in the case of emotional visual stimuli, or at least in the case of facial expressions. An angry face is indeed a threatening stimulus which in itself automatically activates the specific fear system related to defensive mechanisms that have their roots in human evolutionary history (Darwin, 1998; Ohman and Mineka, 2001). Recently, Tipples (2011) found that the magnitude of the overestimation of the presentation duration of angry faces depends on the level of threat experienced by individuals. However, the problem is that the arousal effect produced by the perception of an image of angry face is rapid and short-lived. The biological process of activation therefore occurs very quickly. In a temporal reproduction task, Bar-Haim et al. (2009) showed that the significant temporal overestimation of fearful faces disappeared after $2 \mathrm{~s}$, "as if time perception returns to its baseline state." In these experimental conditions, it is therefore difficult to verify the existence of a multiplicative effect which would support the clock speed hypothesis but would require the use of two duration ranges, one longer than the other, in order to be tested.

The emotional stimuli used in the studies described above therefore have only a limited ability to produce intense forms of emotion which persist over time, with the result that it is only possible to study the effects of emotion on the perception of very short durations. The only studies that have elicited intense, longlived emotions are probably the animal-based studies that have used fear conditioning paradigms based on the administration of electric shocks (e.g., Meck, 1983; Meck and MacDonald, 2007). In the experiment conducted by Meck (1983) using the temporal bisection task, rats were initially trained to discriminate between a short and a long anchor stimulus duration. They were then tested with probe durations (intermediate or similar values) in a footshock and a no-footshock condition. The results showed that the rats responded "long" more often in the footshock condition than in the no-footshock control condition, thus shifting the psychometric function toward the left and lowering the point of subjective equality (bisection point, BP). The BP is the stimulus duration judged to be long and short with equal frequency (proportion of long responses equal to 0.50). Meck (1983) therefore concluded that the footshock stress increased the speed of the animals' internal clock. In a recent study conducted among human adults, Droit-Volet et al. (2010b) employed a similar bisection task with an aversive sound (a 50-ms burst of $95 \mathrm{~dB}$ white noise with instantaneous rise time) heard at the end of the stimulus (blue circle) to be estimated. The emotional effects of this sound had already been tested in the participants on the basis of self-reports and electrophysiological measures (skin conductance response). In line with Meck's results, the authors found that the participants responded more often "long," with a leftward shift of the psychometric function and a lower BP value, on the trials in which the participants expected the aversive sound at the end of the stimulus duration compared to trials in which they expected either a non-aversive sound or no sound. This experiment thus clearly confirms that, in the same way as in animals, fear distorts time perception in human adults by inducing a lengthening effect. This finding is entirely consistent with the works on fear showing that threatening stimuli elicit fear reactions which automatically trigger a wide variety of behavioral and physiological responses (heart rate, muscle contraction), because the organism must be prepared to react as fast as possible for its survival (LeDoux, 2000; Phelps and LeDoux, 2005; Delgado et al., 2006; Phelps, 2006). One of the bodily changes observed in reaction to fear thus consists of a speeding-up of the internal clock (Droit-Volet et al., 2010). However, in these studies, the time distortions were the result of reactions to a detected or expected threatening stimulus which interfered with the processing of time and not the result of the effect of a diffuse emotional state on time judgments in a neutral situation devoid of emotional stimuli. 
The originality of the present study therefore lies in the attempt to examine the effect of mood experience on participants' subsequent time judgment of neutral events. We used an emotion elicitation technique that had not previously been employed in studies of time perception, namely emotional film clips. We used three types of films from the recent database of films validated by Schaefer et al. (2011): films eliciting fear (1) or sadness (2) and neutral control films (3). The selected emotional films were used because they both elicit negative emotions. However, one was high-arousing (frightening film) and the other low-arousing (sad film; Izard, 1991). Before and after the presentation of the films, the participants performed a bisection task. The bisection task administered before the film was designed to provide baseline values. Two duration ranges (200/800 and 400/1600 ms) were used in order to try to identify the mechanisms underlying the effect of emotion on time perception (arousal/multiplicative effect or attention/additive effect) In addition, each participant's mood was assessed at the beginning and the end of each experimental session to identify changes in the mood experience produced by the film and verify whether their mood had dissipated. Our assumption was that after viewing a film, the perception of time would not change when the film was neutral. By contrast, stimulus durations would be judged longer (leftward shift of psychometric function and lower $\mathrm{BP}$ ) after than before the high-arousing, fear-inducing film, and shorter (rightward shift of psychometric function and higher BP) after than before the sadness-inducing film that decreases the arousal level.

\section{MATERIALS AND METHODS PARTICIPANTS}

Forty-five psychology students (Mean age $=19.46, \mathrm{SD}=1.31,40$ women and 5 men) participated in order to fulfill a course requirement and signed a written consent form as required by the Clermont-Ferrand Sud-Est VI Statutory Ethics Committee (CPP).

\section{MATERIALS}

The experiment took place in an isolated room at Blaise Pascal University. The participants were seated in front a PC, with a $17^{\prime \prime}$ screen, that controlled the experimental events via E-prime software. Two loudspeakers were placed on either side of the computer. The stimulus to be timed was always a blue circle presented in the center of the computer screen. The response (long or short) consisted of pressing the " $S$ " and " $L$ " keys of the computer keyboard. The films consisted of excerpts taken from the Schaefer et al. (2011) validated database of emotional films. The films were selected as a function of the discrete emotion elicited (fear, sadness, neutrality) and the intensity of this emotion. The film clips were: The Blair (No. 55, code 65), Scream 1 (No. 16, code 26), and Shining (No. 28, code 38) for the emotion of fear; City of Angels (No. 36, code 46), Philadelphia (No. 62, code 72), and Dangerous Mind (No. 52 code 62), for sadness; and four excerpts of TV weather news and four excerpts of stock market news for the neutral condition. In each emotional condition, two different sequences of film clips were created to form a sequence of $9 \mathrm{~min}$ in length. The direct experience of mood produced in the participants by viewing these films was assessed using the French version of the Brief Mood Introspective Scale (BMIS; Mayer and
Gaschke, 1988) with 18 mood-adjective scales (e.g., calm/calme, nervous/nerveux, aroused/excité, sad/triste, happy/joyeux, frightened/apeuré)(Niedenthal and Dalle, 2001). In the BMIS, the participants rated the intensity of their experience of each of the mood adjectives on a 4-point scale: (XX) definitely do not feel, $(\mathrm{X})$ do not feel, (V) slightly feel, and (VV) definitely feel.

\section{PROCEDURE}

The participants were assigned to one of two duration conditions: $200 / 800$ or $400 / 1600-m s$. In the $200 / 800$ condition, the short $(S)$ anchor stimulus duration was $200 \mathrm{~ms}$ and the long $(L)$ anchor stimulus duration $800 \mathrm{~ms}$. The probe durations were 200, 300, 400, $500,600,700$, and $800 \mathrm{~ms}$. In the $400 / 1600-\mathrm{ms}, S$ and $L$ were 400 and $1600 \mathrm{~ms}$ and the probe durations $400,600,800,1000,1200$, 1400 , and $1600 \mathrm{~ms}$. In each duration condition, the participants completed three emotion sessions as a function of film content (fear, sad, neutral). The sessions were presented with a new random order for each participant, with a 15-min inter-session interval during which the participants were free to leave the experimental room.

Each session consisted of two bisection tasks, a baseline bisection task given before film presentation (pre-film), which was followed by a test bisection task administered after film presentation (post-film). In the bisection task performed before the film, the participants were first presented with $S$ and $L$ once. They then performed the bisection task with eight blocks of seven trials (56 trials), with one trial being administered for each probe duration. For each probe duration, they therefore had to indicate whether it was more similar to $S$ or to $L$ by pressing the corresponding button. The durations within each block were presented randomly. The inter-trial interval was also randomly chosen between 500 and $1500 \mathrm{~ms}$. The participants started the trial after the word "ready/prêt" was presented, immediately followed by a $200-\mathrm{ms}$ interval and then the stimulus to be timed (blue circle). The procedure used for the post-film bisection task was exactly the same as that used in the pre-film bisection task, except that it was divided into two series of four blocks of trials, with emotional films being presented for $9 \mathrm{~min}$ before each series of blocks. In addition, the participants completed the BMIS at the start and end of each session, i.e., before and after viewing the emotional films.

\section{RESULTS}

\section{ASSESSMENT OF EMOTIONS}

Table 1 presents the mean mood ratings expressed by the participants on the 4-point scales for the 18 adjectives of the BMIS that was completed just before the pre-film bisection task and after the post-film bisection task. For each emotional condition taken separately, viewing the emotional films significantly changed the participants' mood as indicated by the overall ANOVAs on mood experience ratings with mood adjectives and the bisection task (before vs. after the film) as within-subjects factors. There was indeed a significant interaction between the mood adjectives and the bisection phases in each emotional condition [Fear, $F(17,748)=19.74$, Sadness, $F(17,748)=15.52$, and Neutral, $F(17,748)=8.19, p<0.05]$. As shown in Table 1 , in the fear condition, the participants felt less calm and more aroused or nervous after than before watching frightening movies [paired 
Table 1 | Mean rate $(M)$ and SEM of experienced mood on 4-point scales for 18 mood adjectives of the French version of the Brief Mood Introspective Scale (BMIS) given before and after the film, for each emotional content of the films (fear, sadness, and neutral).

\begin{tabular}{|c|c|c|c|c|c|c|c|c|c|c|c|c|}
\hline & \multicolumn{4}{|c|}{ Fear } & \multicolumn{4}{|c|}{ Sadness } & \multicolumn{4}{|c|}{ Neutral } \\
\hline & \multicolumn{2}{|c|}{ Before } & \multicolumn{2}{|c|}{ After } & \multicolumn{2}{|c|}{ Before } & \multicolumn{2}{|c|}{ After } & \multicolumn{2}{|c|}{ Before } & \multicolumn{2}{|c|}{ After } \\
\hline Lively & 2.44 & 0.12 & 2.42 & 0.13 & 2.49 & 0.14 & $2.07 * *$ & 0.13 & 2.53 & 0.13 & $2.09 * *$ & 0.13 \\
\hline Happy & 2.78 & 0.11 & $2.44^{* *}$ & 0.11 & 2.87 & 0.12 & $2.4^{* *}$ & 0.10 & 2.84 & 0.12 & $2.6^{* *}$ & 0.12 \\
\hline Caring & 2.47 & 0.12 & 2.42 & 0.12 & 2.51 & 0.11 & $2.31 *$ & 0.12 & 2.6 & 0.11 & $2.4^{*}$ & 0.12 \\
\hline Content & 2.76 & 0.12 & $2.22 * *$ & 0.11 & 2.78 & 0.12 & $2.24 * *$ & 0.12 & 2.8 & 0.12 & 2.69 & 0.13 \\
\hline Gloomy & 1.4 & 0.11 & 1.58 & 0.12 & 1.31 & 0.09 & $2^{* *}$ & 0.13 & 1.53 & 0.12 & 1.51 & 0.11 \\
\hline Aroused & 1.58 & 0.09 & $1.96 * *$ & 0.13 & 1.78 & 0.10 & $1.31 * *$ & 0.07 & 1.62 & 0.12 & 1.53 & 0.11 \\
\hline Drowsy & 1.96 & 0.14 & $1.6^{*}$ & 0.11 & 1.87 & 0.14 & 2.04 & 0.14 & 1.93 & 0.14 & $2.56^{* *}$ & 0.14 \\
\hline Calm & 3.27 & 0.11 & $1.82 * *$ & 0.11 & 3.13 & 0.12 & 3.11 & 0.11 & 2.98 & 0.12 & 3.07 & 0.12 \\
\hline Loving & 2.62 & 0.14 & $2.16^{* *}$ & 0.14 & 2.58 & 0.14 & 2.53 & 0.13 & 2.58 & 0.14 & $2.31 *$ & 0.15 \\
\hline Fedup & 1.4 & 0.10 & 1.73 & 0.14 & 1.47 & 0.13 & 1.4 & 0.11 & 1.38 & 0.10 & $1.96 * *$ & 0.15 \\
\hline Active & 1.96 & 0.12 & $2.29 *$ & 0.12 & 2.11 & 0.12 & $1.78^{* *}$ & 0.11 & 1.87 & 0.13 & $1.67^{*}$ & 0.11 \\
\hline Worried & 1.31 & 0.10 & $2.09 * *$ & 0.13 & 1.38 & 0.10 & $1.58^{*}$ & 0.11 & 1.44 & 0.10 & $1.29^{*}$ & 0.08 \\
\hline Frightened & 1.11 & 0.05 & $2.22 * *$ & 0.14 & 1.09 & 0.04 & 1.18 & 0.07 & 1.09 & 0.07 & 1.14 & 0.03 \\
\hline
\end{tabular}

${ }^{*}$ Difference between the base line and the test significant at $p<05$, and ${ }^{*}$ at $p<0.01$.

sample $t$-test, $t(44)=9.57, t(44)=2.78, t(44)=5.144$, respectively, all $p<0.05]$. They also reported being more frightened and worried $[t(44)=7.77, t(44)=5.48, p<0.05]$. Watching sad films also caused the participants to feel sadder and gloomier and less happy $[t(44)=8.17, t(44)=6.05, t(44)=5.72, p<0.05]$. This also reduced the feeling of arousal $[t(44)=3.32, p<0.05]$, although the participants reported that they remained calm between the two bisection tasks $[t(44)=0.19, p>0.05]$. Neutral films also changed the individuals' moods. However, it affected neither the arousal and calmness dimensions $[t(44)=0.68$, $t(44)=0.71, p>0.05]$, nor the specific emotions of fear and sadness $[t(44)=1.21, t(44)=1.83, p>0.05]$. It simply made the participants feel more tired, less lively and less happy $[t(44)=3.08$, $t(44)=3.67, t(44)=2.88$, respectively, $p<0.05$ ]. In other words, they were more dispirited when they completed their reports, $t(44)=3.83, p<0.05$. In sum, watching the emotional films elicited the expected discrete emotions, i.e., fear and sadness, in the participants. These emotions were associated with an increase and a decrease in arousal level respectively, although the magnitude of the difference in arousal between the pre- and post-film bisection tasks was larger for the frightening films.

\section{TEMPORAL BISECTION}

For each emotional condition (fear, sadness, neutral), the psychometric functions were represented by plotting the proportion of long responses $[p$ (long)] against the comparison durations in the $200 / 800$ and the $400 / 1600-m s$ duration range for the two bisection tasks, i.e., before and after the presentation of the films (Figures 1-3). To account for the leftward or the rightward shift

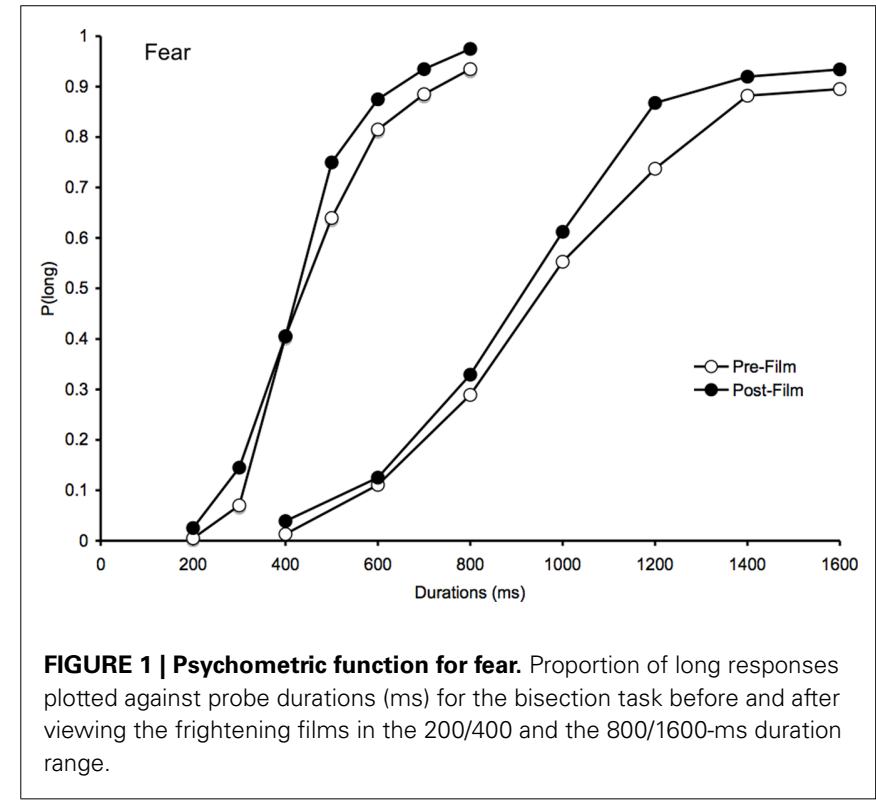

of the psychometric function for the post-film bisection task compared to that for the pre-film bisection task, we calculated the BP. As reported in introduction, the $\mathrm{BP}$ is the point of subjective equality, $[t(p$ long $)=0.50]$. A decrease in the BP value reveals a time distortion in the form of a lengthening effect which is consistent with a leftward shift of the psychometric function. In contrast, an increase in the BP indicates a shortening effect associated with a 


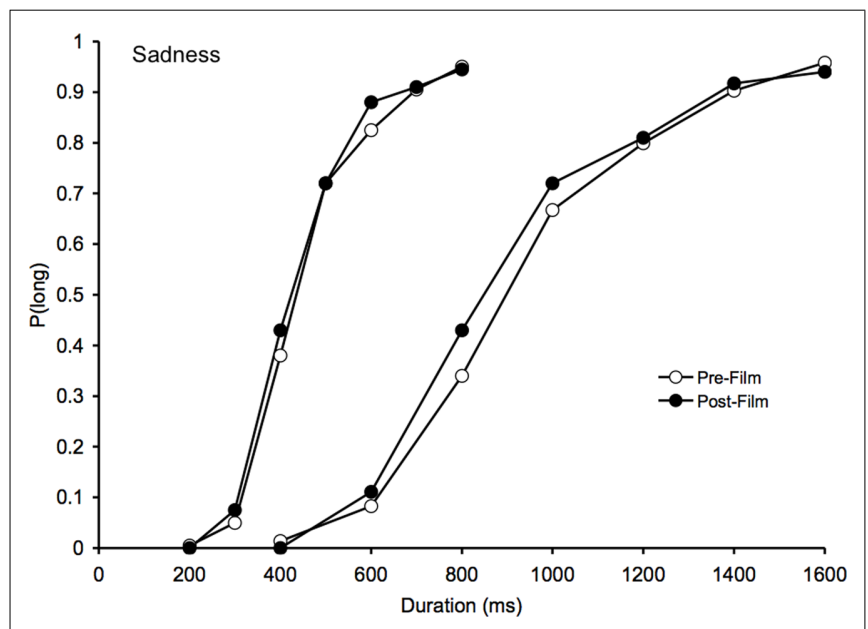

FIGURE 2 | Psychometric function for sadness. Proportion of long responses plotted against probe durations (ms) for the bisection task before and after viewing the sad films in the 200/400 and the 800/1600-ms duration range.

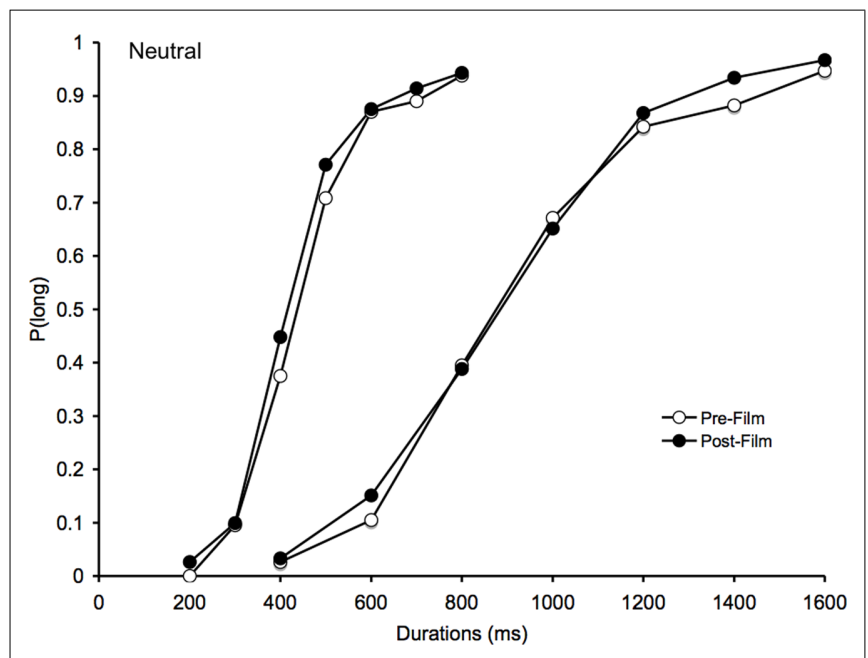

FIGURE 3 | Psychometric function for neutral. Proportion of long responses plotted against comparison durations (ms) for the bisection task before and after viewing the neutral films in the 200/400 and the 800/1600-ms duration range.

rightward shift of the psychometric function. This measures was derived from the slope and intercept parameters obtained by fitting a logistic function to the individual subject data. In the case of one participant, the fit was not significant in any emotional condition, and it was not significant in one of the three conditions for three other participants. The results for these participants were therefore excluded from the subsequent analyses. Table 2 presents the BP obtained in this way.

An initial omnibus ANOVA was performed on the BP with duration range as between-subjects factor (200/800 vs. 400/1600) and two within-subjects factors: (1) the bisection task (preand post-film) and (2) the emotion (fear, sadness, neutral). The
Table 2 | Mean (M) and SEM for bisection point (BP) and Weber ratio (WR) for the films inducing fear and sadness as well as for the neutral films before and after viewing the films.

\begin{tabular}{|c|c|c|c|c|}
\hline & \multicolumn{4}{|c|}{ Fear } \\
\hline & \multicolumn{2}{|c|}{ BP } & \multicolumn{2}{|c|}{ WR } \\
\hline & $M$ & SEM & $M$ & SEM \\
\hline \multicolumn{5}{|l|}{$200 / 800$} \\
\hline Pre-film & 494 & 34 & 0.20 & 0.05 \\
\hline Post-film & 446 & 35 & 0.14 & 0.03 \\
\hline \multicolumn{5}{|l|}{$400 / 1600$} \\
\hline Pre-film & 1045 & 38 & 0.17 & 0.05 \\
\hline \multirow[t]{4}{*}{ Post-film } & 910 & 39 & 0.17 & 0.03 \\
\hline & \multicolumn{4}{|c|}{ Sadness } \\
\hline & \multicolumn{2}{|c|}{ BP } & \multicolumn{2}{|c|}{ WR } \\
\hline & $M$ & SD & $M$ & SD \\
\hline \multicolumn{5}{|l|}{$200 / 800$} \\
\hline Pre-film & 499 & 27 & 0.14 & 0.03 \\
\hline Post-film & 481 & 31 & 0.16 & 0.03 \\
\hline \multicolumn{5}{|l|}{$400 / 1600$} \\
\hline Pre-film & 996 & 31 & 0.12 & 0.03 \\
\hline \multirow[t]{4}{*}{ Post-film } & 964 & 37 & 0.12 & 0.03 \\
\hline & \multicolumn{4}{|c|}{ Neutral } \\
\hline & \multicolumn{2}{|c|}{ BP } & \multicolumn{2}{|c|}{ WR } \\
\hline & $M$ & SD & $M$ & SD \\
\hline \multicolumn{5}{|l|}{$200 / 800$} \\
\hline Pre-film & 483 & 25 & 0.15 & 0.02 \\
\hline Post-film & 462 & 28 & 0.14 & 0.03 \\
\hline \multicolumn{5}{|l|}{$400 / 1600$} \\
\hline Pre-film & 949 & 28 & 0.15 & 0.02 \\
\hline Post-film & 918 & 31 & 0.20 & 0.03 \\
\hline
\end{tabular}

ANOVAs on $p$ (long) are not presented because the results were similar to those found for the BP. The ANOVA on the BP suggested the presence of time distortions as a function of task, i.e., pre- or post-film, and emotion. Indeed, there was a significant interaction between the timing of the task and the emotion, $F(2,80)=3.24, p<0.05$, which subsumed a significant task effect, $F(1,40)=13.91, p<0.05$, and no significant emotion effect, $F(2$, $80)=0.63, p>0.05$. The effect of duration range was also significant, $F(1,40)=228.99, p<0.05$, but duration range did not interact with any other factors (all $p>0.05$ ). In the case of the pre-film bisection task, no significant effect of emotion, $F(2$, $82)=1.19, p>0.05$, was found, thus indicating that there were no differences between the emotion conditions before the participants viewed the films. Consequently, to examine how the mood induced by a type of film modifies time perception, we compared the bisection performance before the film with that after the film for each emotional film taken separately. To do this we 
ran an ANOVA on the BP with task time as within-subjects factor and duration range as a between-subjects factor for each type of emotional induction.

For the fear-inducing film (Figure 1), there was a clear leftward shift of the psychometric function after the film compared to the psychometric function before the film. This suggests that the comparison durations were judged longer after the participants had watched fear-inducing films that increased their arousal levels as the BMIS scores indicated. In line with this leftward shift of the psychometric functions, the ANOVA on the BP showed a significant main effect of task, $F(1,41)=20.05, p<0.05$, indicating that the BP was lower after than before the frightening film (Table 2). There was also a significant task $\times$ duration interaction, $F(1,41)=4.55, p<0.05$, which subsumed a significant main effect of duration, $F(1,41)=110.75, p<0.05$. For each duration range, the BP was indeed lower after than before the film [200/400, $F(1$, $23)=9.55 ; 800 / 1600, F(1,18)=10.52$, both $p<0.05]$. The probe durations were thus judged longer after the frightening films, when the participants had experienced fear and were more aroused or nervous. In line with this suggestion, for the post-film bisection task, there was a significant correlation between the BP value and the subjective individual feeling of being more nervous/nerveux after viewing the frightening films $(R=0.32, p<0.05)$ : The more nervous the participants reported being in the BMIS after the film, the smaller their BP value was. The correlation between the BP value and the aroused/excité adjective did not reach significance, probably related to the French word "excité" that did not verbally describe as well as the word "nerveux" the subjective mood experienced by the participants after viewing a frightening film. In addition, and in accordance with the hypothesis of a clock rate mechanism (multiplicative effect), the magnitude of the difference in the BPs between the pre-film and post-film bisection tasks was larger for the long than for the shorter duration range. When we calculated the difference in the BPs between these two bisection tasks, the one-way ANOVA did indeed reveal a significant effect of duration that indicates that the task-related difference was larger in the $400 / 1600$ than in the $200 / 800$-ms duration condition [ 133.65 vs. $43.38, F(1,41)=4.55, p<0.05$ ]

With regard to the sad film clips, the assessment of mood experience using the BMIS suggested that these films did indeed induce sadness in the participants and reduce their arousal level. However, as Figure 2 suggests, this change in mood did not significantly modify the participants' time perception. The ANOVA on the BP found neither a significant effect of task, $F(1,41)=2.08, p>0.05$, nor any significant task $\times$ duration interaction, $F(1,41)=0.09$, $p>0.05$. There was only a significant main effect of duration, $F(1$, $41)=163.72, p<0.05$, thus indicating that the BP value was higher in the $400 / 1600$ than in the $200 / 800-m s$ duration condition.

As expected, for the neutral films, the psychometric functions obtained after the neutral films were also similar to those obtained before the neutral films (Figure 3). Consequently, the effect of task on the BP was not significant $[F(1,41)=2.08, p>0.05]$, and neither was the effect of the task $\times$ duration interaction $[F(1$, $41)=1.44, p>0.05]$. There was only a significant main effect of duration for the BP, $F(1,41)=163.72, p>0.05$.

We performed the same analyses on the Weber ratio (WR; Table 2), which is an index of sensitivity to time. It is the Difference
Limen $[t(p$ long $)=0.75-t(p($ long $)=0.25) / 2]$ divided by the BP. A high WR value indicates that participants' temporal discrimination varies and that they find it more difficult to discriminate between two durations. The omnibus ANOVA on the WR did not show any significant effect (all $p>0.05$ ). This is verified in the ANOVA conducted for the sad film [task, $F(1,41)=0.06$, duration, $F(1,41)=1.29$, task $\times$ duration, $F(1,41)=0.057$, all $p>0.05]$ and for the neutral film [task, $F(1,41)=1.11$, duration, $F(1,41)=1.05$, task $\times$ duration $F(1,41)=1.44$, all $p>0.05]$. This indicates that Weber's Law continued to hold and that the sensitivity to time did not vary after viewing the neutral film and the sad films despite the reduction in arousal reported in the BMIS questions for the sadness.

For the fear-inducing film, in line with the general ANOVA, the ANOVA on the WR showed neither a significant main effect of emotion, $F(1,41)=1.11, p>0.05$, or of duration, $F(1,41)=0.02$, $p>0.05$, nor any significant emotion $\times$ duration interaction, $F(1$, $41)=1.12, p>0.05$. This suggests that the scalar property of time continued to apply, with a constant WR being observed across the different durations. However, for the frightening film associated with a leftward shift of the psychometric function, this also confirms that the leftward shift of the psychometric functions in response to fear was proportional (multiplicative) rather than absolute (additive) in nature. This proportional shift was confirmed by the good superimposition of the psychometric functions when plotted on a relative scale by dividing the probe durations by the mean group BP (Figure 4). Overall, these different results suggest that, in the present experiment which used emotional films, the internal clock system ran faster when the participants experienced a fearful and arousing mood that caused the stimulus durations to be judged longer.

\section{DISCUSSION}

The originality of the present study lies in the fact that it attempts to investigate the effect of mood experience on the time perception of neutral events rather than to investigate the estimation of the duration of emotional events. We therefore showed our participants validated emotional films that lasted for long periods $(9 \mathrm{~min})$ in order to modify their general mood. Our results demonstrated that viewing emotional films for periods of several

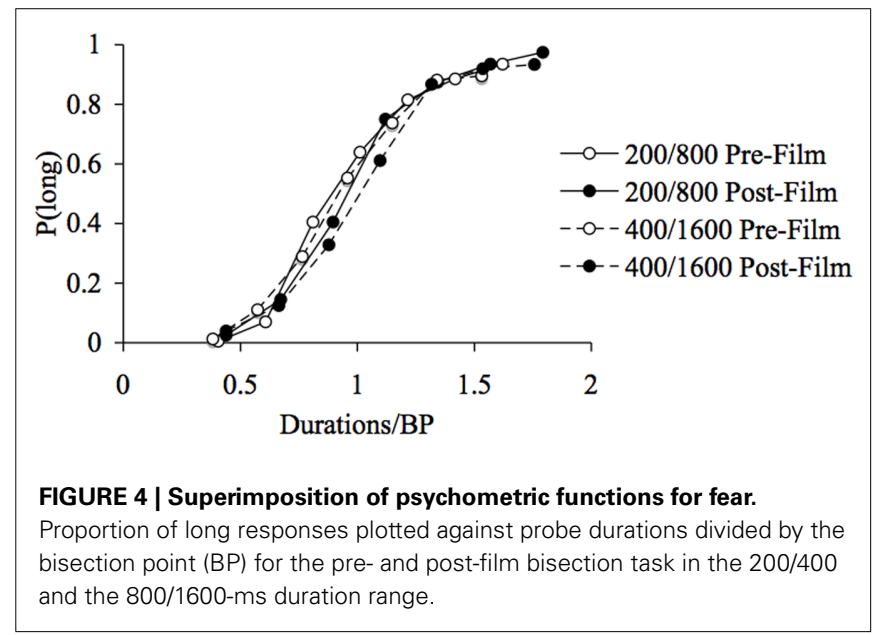


minutes changed the participants' mood, and that the mood experience may affect the perception of time in a subsequent temporal task. Three types of emotional films were used: frightening, sad, and neutral control films. In line with other studies which have used emotional films (Rottenberg et al., 2007), our results showed that viewing the frightening films increased the participants' feeling of fear and their arousal level. In contrast, viewing sad films induced sadness and decreased the level of arousal, while viewing neutral films did not significantly change the arousal dimension or the discrete emotions. The participants were simply bored by watching excerpts of TV weather or stock market news. However, and more originally, our results showed that the mood elicited by the films affected time judgments in a subsequent temporal task in which no emotional stimuli were present. The stimulus to be timed was simply a blue circle of the type often used in studies of time perception in humans. However, our results showed that the perception of time was distorted only following the induction of fear rather than of sadness.

Our study showed that the mood of fear produced by the frightening films had a lengthening effect on time perception, with the result that the stimulus durations were judged longer after than before viewing these emotional films. Indeed, our results indicated that the psychometric functions were shifted toward the left in the post-compared to the pre-film bisection tasks, with a significant lowering of the BP being observed. In addition, our results demonstrated that the fear-related effect on the perception of time was not due to simply watching a film, independently of the elicited emotion. Indeed, the neutral films did not affect time perception. In sum, a clear distortion of time was produced by the mood of fear induced by the films. Furthermore, this time distortion was not associated with an impairment of temporal discrimination. Indeed, our findings showed that variability in time discrimination did not change between the experimental conditions as indicated by the fact that the WR values were similar in the different conditions. They also showed that Weber's law holds whatever the emotional condition, with a constant WR being observed for the different stimulus duration ranges. Overall, these results are entirely consistent with those found in temporal studies using other negative highly arousing emotional stimuli (aversive sounds, faces, pictures; for a recent review, see Droit-Volet, in press). As in these earlier studies, we may thus assume that the lengthened time experience observed in response to the frightening films in our study was due to the automatic activation of the nervous system by the emotion of fear. Since the internal clock system depends on the dynamic functioning of the brain, when its rate increases in response to the emotion of fear, it produces more time units for the representation of time, and time is judged longer. In line with this idea, our results suggested a significant correlation between the subjective individual feeling of being more nervous after viewing the frightening films, on the one hand, and the magnitude of the lengthening effect, on the other. The more nervous the participants reported feeling after the frightening films, the lower the associated $\mathrm{BP}$ values were. According to the mathematics of the internal clock models, a clock rate-based mechanism requires a multiplicative effect in which the magnitude of the temporal overestimation is proportional to the duration values, i.e., it is greater for the long than for the shorter stimulus durations. This is confirmed by our data that showed a larger leftward shift of the psychometric function in response to fear in the $400 / 1600$ than in the $200 / 800-\mathrm{ms}$ duration conditions, and a greater difference between the BP values in the pre- and post-film bisection tasks in the long than in the shorter duration conditions. Furthermore, in the fear condition, when the psychometric functions for the two duration ranges were plotted against a relative scale (Probe durations/BP), they superimposed well. Overall, these different results support the idea that viewing frightening films speeds up the internal clock system and consequently lengthens the experience of time.

Unlike the emotion of fear, sadness did not modify time perception in the present bisection task despite the fact that the participants reported being sadder and less aroused after than before viewing the sad films in the BMIS. The mood of sadness is considered to be a low-arousal emotion inducing a slowing down of mental and motor activity (Izard and Ackerman, 2000). Consequently, on the basis of our results, we can suggest that a low-arousal emotion, i.e., an emotion that is not arousing, does not affect time perception. This is consistent with studies of emotions that have found that the durations of low-arousal stimuli were less overestimated that those of high arousal stimuli (Angrilli et al., 1997). Using negative low-arousal pictures from the IAPS, Gil and Droit-Volet (submitted) recently found that negative low-arousal pictures had few effect on time estimation compared to neutral pictures. Nevertheless, we might have expected that the decrease in arousal after the sad film would result in a slowing down of the internal clock system that, in turn, would have shortened the perceived duration. It is possible that, in our experiment, the magnitude of the decrease in arousal in the participants when viewing the sad films was insufficient to produce a significant physiological slowing down of the internal clock system. This type of slowing down may be observed to a greater extent during a major depressive episode associated with a real loss of energy (Hoffer and Osmond, 1962; Wyrick and Wyrick, 1977; Gil and Droit-Volet, 2009) or in response to the administration of sedative drugs (e.g., Rammsayer, 1989; Lustig and Meck, 2005). In a laboratory situation, it is therefore difficult to slow down the internal clock using external stimuli that significantly affect temporal behavior. Although several studies have used periodic clicks to accelerate the internal clock with click rates of between 5 and $25 \mathrm{~Hz}$ (Treisman et al., 1990; Ortega and López, 2008; Wearden, 2008), none have successfully attempted to slow down the internal clock by using lower click frequencies. In addition, these click rate effects have been observed in "relative" conditions, in which the clock slows down after having been accelerated, rather than in an "absolute" condition, i.e., following the measurement of a baseline clock rate. In this latter condition, Wearden (2008) was nevertheless able to manipulate the slowing down of the internal clock by designing a particularly boring experiment with long intervals between each trial. In these specific conditions, a significant rightward shift of time estimates was observed in the boring compared but to the non-boring experiment in both a temporal generalization and a temporal verbal estimation task but not in a temporal bisection task. In sum, it is very difficulty, but not impossible, to slow down the general nervous system and its internal clock by means of external emotional stimuli. 
This difficulty in finding emotional stimuli that can slow down the internal clock system is due to the fact that human beings are genetically predisposed to produce automatic behavioral reactions rather than to reduce their rate of action when events occur in the environment. The motivation for action is thus one of the main factors explaining the acceleration of the internal clock mechanisms in response to emotion. Time distortions thus indirectly allow us to identify the fundamental adaptive function of emotions (Frijda, 1986). Fear is an emotional state of readiness for action. As argued by Droit-Volet (in press), a threatening stimulus triggers a cascade of physiological reactions: the pupils dilate, the heart accelerates, blood pressure increases, the muscles contract. The whole body is mobilized to react, i.e., to escape or to attack as quickly as possible. Consequently, without disrupting the processing of time, the internal clock runs faster, thus ensuring that the individual is prepared to react earlier to incoming events. In contrast, sadness is a poorly understood emotion, an obscure emotion in Lazarus' (1991) words. Its function is not clearly identified, all the more so since it often co-exists alongside other emotions (guilt, anger). In addition, the nature of the feeling of sadness depends on its source (dull pain, grief, frustration, empathy with others), as well as on its intensity and duration, with prolonged periods of sadness resulting in depression (Freed and Mann, 2007). Nevertheless, sadness is thought to be "linked to a loss, such as the death of someone we love, the failure of a central life value or role, or the loss of the positive regard of another" (Lazarus, 1991, p. 247). This loss therefore involves a certain degree of disengagement. In line with this behavioral description of emotions, neuroscientific studies using neuroimaging techniques have

\section{REFERENCES}

Angrilli, A., Cherubini, P., Pavese, A., and Manfredini, S. (1997). The influence of affective factors on time perception. Percept. Psychophys. 59, 972-982.

Bar-Haim, Y., Kerem, A., Lamy, D., and Zakay, D. (2009). When time slow down: the influence of threat on time perception in anxiety. Cogn. Emot. 24, 1-9.

Barrett, L. F. (2006). Are emotion natural kinds. Assoc. Psychol. Sci. 1, 28-57.

Bradley, M. M., and Lang, P. J. (1999). International Affective Digitized Sounds (IADS): Stimuli, Instructions Manual and Affective Ratings. (Tech. Rep. No. B-2). Gainesville, FL: University of Florida, Center for Research in Psychophysiology.

Coan, J. A., and Allen, J. J. B. (2007). Handbook of Emotion Elicitation and Assessment. Oxford: Oxford University Press.

Coull, J. T., Cheng, R. K., and Meck, W. H. (2011). Neuroanatomical and neurochemical substrates of timing. Neuropsychopharmacology 36, 3-25.

Darwin, C. (1998). The Expression of the Emotions in Man and Animals. Oxford: Oxford University
Press. [Original work published 1872].

Delgado, M. R., Olsson, A., and Phelps, E. A. (2006). Extending animal models of fear conditioning to humans. Biol. Psychol. 73, 39-48.

Doi, H., and Shinohara, K. (2009). The perceived duration of emotional face is influenced by the gaze direction. Neurosci. Lett. 457, 97-100.

Droit-Volet, S., Bigand, E., Ramos, D., and Bueno, J. L. O. (2010a). Time flies with music whatever its emotional valence. Acta Psychol. (Amst.) 135, 226-236.

Droit-Volet, S., Mermillod, M., Cocenas-Silva, R., and Gil, S. (2010b). The effect of expectancy of a threatening event on time perception in human adults. Emotion 10, 908-914.

Droit-Volet, S., Brunot, S., and Niedenthal, P. M. (2004). Perception of the duration of emotional events. Cogn. Emot. 18, 849-858.

Droit-Volet, S., and Gil, S. (2009). The time-emotion paradox. J. Philos. Trans. R. Soc. Lond. B Biol. Sci. 364, 1943-1953.

Droit-Volet, S., and Meck, W. H. (2007). How emotions colour our time perception. Trends Cogn. Sci. (Regul. Ed.) 11, 504-513.

shown a clear relationship between the emotion of fear, the amygdala of the basal ganglia, and the dopaminergic system (LeDoux, 2005). The dopaminergic system is known to be involved in the detection of incoming events that are potentially dangerous and lead to a specific motor reaction. As Nieoullon and Coquerel (2003) explained, Dopa is a gatekeeper allowing stimuli to access high-order voluntary motor regions of the brain. More specifically, as reported above, time judgments have been shown to be highly sensitive to arousal effects and to the administration of dopaminergic drugs. In contrast, except in the case of major depression, little is known in the neuroscientific domain about episodes of sadness lasting several minutes. In the case of sadness, it seems that no specific neuroanatomical structures are involved, although the induction of a sad mood seems to involve the anterior cingulate cortex (Barrett, 2006; Freed and Mann, 2007). This probably explains why sadness had no effect on time in the present study. Whatever the case, the perception of time in the presence of sadness is particularly intriguing and various exploratory experiments are now required in order to gain a better understanding of individuals' relation to time in the emotional state of sadness.

\section{ACKNOWLEDGMENTS}

This work was supported by the European Cooperation in Science and Technology (COST; TIMELY) and by a Partner University Fund between France and United-States on Emotion and Time. It was also supported by a grant from the Agence Nationale de la recherche (ANR) Emotion(s), Cognition, Comportement from France.

Droit-Volet, S., and Wearden, J. H. (2002). Speeding up an internal clock in children? Effects of visual flicker on subjective duration. Q. J. Exp. Psychol. 55B, 193-211.

Droit-Volet, S. (in press). "What emotions tell us about time," in Subjective Time: The Philosophy, Psychology, and Neuroscience of Temporality, eds D. Llyod and V. Arstila (Cambridge, MA: MIT Press).

Durstewitz, D. (2004). Neural representation of interval time. Neuroreport $15,745-749$.

Effron, D. A., Niedenthal, P. M., Gil, S., and Droit-Volet, S. (2006). Embodied temporal perception of emotion. Emotion 6, 1-9.

Falk, J. L., and Bindra, D. (1954). Judgment of time as a function of serial position and stress. J. Exp. Psychol. 39, 327-331.

Freed, P. J., and Mann, J. J. (2007). Sadness and loss: toward a neurobiopsychosocial model. Am. J. Psychiatry $164,1,28-34$.

Frijda, N. H. (1986). The Emotions. New York: Cambridge University Press.

Gibbon, J., Church, R. M., and Meck, W. (1984). "Scalar timing in memory," in Annals of the New York Academy of Sciences, 423: Timing and Time Perception, eds J.
Gibbon and L. Allan (New York: New York Academy of Sciences), 52-77.

Gil, S., and Droit-Volet, S. (2009). Time perception, depression and sadness. Behav. Processes 80, 169-176.

Gil, S., and Droit-Volet, S. (2011a) Time flies in the presence of angry faces, depending on the temporal task used! Acta Psychol. (Amst.) 136, 354-362.

Gil, S., and Droit-Volet, S. (2011b). Time perception in response to ashamed faces in children and adults. Scand. J. Psychol. 52, 138-145.

Gil, S., Niedenthal, P. M., and DroitVolet, S. (2007). Anger and time perception in children. Emotion 7, 219-225.

Grommet, E. K., Droit-Volet, S., Gil, S., Hemmes, N. S., Baker, A. H., and Brown, B. (2010). Effects of a fear cue on time estimation in human observers. Behav. Processes 86, 88-93.

Hoffer, A., and Osmond, H. (1962). The relationship between mood and time perception. Psychiatr. Q. Suppl. 36, 87-92.

Izard, C. E. (1991). The Psychology of Emotions. New York: Plenum. 
Izard, C. E., and Ackerman, B. (2000). Motivational, Organizational and Regulatory Functions of Discrete Emotions. Handbook of Emotions, 2nd Edn. New York: Guilford Press.

Karmarkar, U. R., and Buonomano, D. V. (2007). Timing in the absence of clocks: encoding time in neural network states. Neuron 53, 427-438.

Lang, P. J., Bradley, M. M., and Cuthbert, B. N. (2008). International Affective Picture System (IAPS): Affective Ratings of Pictures and Instruction Manual. Technical Report A8. Gainesville, FL: University of Florida.

Langer, J., Wapner, S., and Werner, H. (1961). The effect of danger upon the experience of time. Am. J. Psychol. 74, 94-97.

Lazarus, R. S. (1991). Emotion and Adaptation. New York: Oxford University Press.

LeDoux, J. E. (2000). Emotion circuits in the brain. Annu. Rev. Neurosci. 23, 155-184.

LeDoux, J. E. (2005). Fear and the brain. Where have we been and where are we going. Biol. Psychiatry 44, 1229-1238.

Lee, K-H., Seelam, K., and O'Brien, T. (2011). The relativity of time perception produced by facial emotion stimuli. Cogn. Emot. doi:10.1080/02699931.2010.544455. [Epub ahead of print].

Lustig, C., and Meck, W. H. (2005). Chronic treatment with haloperidol induces deficits in working memory and feedback effects of interval timing. Brain Cogn. 58, 9-16.

Maricq, A. V., Roberts, S., and Church, R. M. (1981). Methamphetamine and time estimation. J. Exp. Psychol. Anim. Behav. Process. 7, 18-30.

Matell, M. S., and Meck, W. H. (2004). Cortico-striatal circuits and interval timing: coincidence-detection of oscillatory processes. Brain Res. Cogn. Brain Res. 21, 139-170.

Mayer, J. D., and Gaschke, Y. N. (1988). The experience and meta-experience of mood. J. Pers. Soc. Psychol. 55, 102-111.

Meck, W. H. (1983). Selective adjustment of speed of internal clock and memory processes. J. Exp. Psychol. Anim. Behav. Process. 9, 171-201.

Meck, W. H., and MacDonald, C. J. (2007). Amygdala inactivation reverses fear's ability to impair divided attention and make time still. Behav. Neurosci. 121, 707-720.

Mella, N., Conty, L., and Pouthas, V. (2011). The role of physiological arousal in time perception: psychophysiological evidence from an emotion regulation paradigm. Brain Cogn. 75, 2, 182-187.

Niedenthal, P. M., and Dalle, N. (2001). Le mariage de mon meilleur ami: emotional response categorization during naturally-induced emotional states. Eur. J. Soc. Psychol. 31, 737-742.

Nieoullon, A., and Coquerel, A. (2003). Dopamine: a key regulator to adapt action, emotion, motivation and cognition. Curr. Opin. Neurol.16, 3-9.

Noulhiane, M., Mella, N., Samson, S., Ragot, R., and Pouthas, V. (2007). How emotional auditory stimuli modulate time perception. Emotion 7, 697-704.

Ohman, A., and Mineka, S. (2001). Fears, phobias, and preparedness. Toward an evolved module of fear and fear learning. Psychol. Rev. 108, 483-522.

Ortega, L., and López, F. (2008). Effects of visual flicker on subjective time in a temporal bisection task. Behav. Processes 78, 380-386.

Penney, T., Gibbon, J., and Meck, W. (2000). Differential effects of auditory and visual signals on clock speed and temporal memory. J. Exp. Psychol. Hum. Percept. Perform. 26, 1770-1787.

Penton-Voak, I. S., Edwards, R., Percival, K., and Wearden, J. H. (1996). Speeding up an internal clock in humans? Effects of click trains on subjective duration. J. Exp. Psychol. Anim. Behav. Process. 22, 307-320.

Peretz, I., Gagnon, L., and Bouchard, B. (1998). Music and emotion: perceptual determinants, immediacy, and isolation after brain damage. Cognition 68, 111-141.

Phelps, E. A. (2006). Emotion and cognition: insights from studies of the human amygdala. Annu. Rev. Psychol. 57, 27-53.

Phelps, E. A., and LeDoux, J. E. (2005). Contributions of the amygdala to emotion processing: from animal models to human behavior. Neuron 48, 175-187.

Rammsayer, T. (1989). Dopaminergic and serotoninergic influence on duration discrimination and vigilance. Pharmacopsychiatry 22, 39-43.

Rammsayer, T. (2009). Effects of pharmacologically induced dopaminereceptor stimulation on human temporal information processing. $\mathrm{Neu}$ roquantology 7, 103-113.

Rottenberg, J., Ray, R. B., and Gross, J. J. (2007). "Emotion eliciting films," in Handbook of Emotion Elicitation and Assessment, eds J. A. Coan and J. J. B. Allen (Oxford: Oxford University Press), 9-28.

Schaefer, A., Nils, F., Sanchez, X., and Philippot, P. (2011). Assessing the effectiveness of a large database of emotion-eliciting films: a new tool for emotion researchers. Cogn. Emot. $24,1153-1172$.

Thayer, S., and Schiff, W. (1975). Eyecontacts, facial expression, and the experience of time. J. Soc. Psychol. 95 117-124.

Tipples, J. (2008). Negative emotionality influences the effects of emotion on time perception. Emotion 8, 127-131.

Tipples, J. (2011).When time stands still: fear-specific modulation of temporal bias due to threat. Emotion 11, 74-80.
Treisman, M. (1963). Temporal discrimination and the indifference interval: implications for a model of the "internal clock." Psychol. Monogr 77, 1-13.

Treisman, M., Faulkner, A., Naish, P. L. N., and Brogan, D. (1990). The internal clock: evidence for a temporal oscillator underlying time perception with some estimates of its characteristic frequency. Perception 19, 705-748.

Van Wassenhove, V., Wittmann, M., Craig, A. D., and Paulus, M. P. (2011). Psychological and neural mechanisms of subjective time dilation. Front. Neurosci. 5:56. doi: 10.3389/fnins.2011.00056

Wearden, J. H. (2008). Slowing down an internal clock: implications for accounts of performance on four timing. Q. J. Exp. Psychol. 61, 263-274.

Wyrick, R. A., and Wyrick, L. C. (1977). Time experience during depression. Arch. Gen. Psychiatry 34, 1441-1443.

Conflict of Interest Statement: The authors declare that the research was conducted in the absence of any commercial or financial relationships that could be construed as a potential conflict of interest.

Received: 30 May 2011; paper pending published: 16 June 2011; accepted: 22 July 2011; published online: 09 August 2011. Citation: Droit-Volet S, Fayolle SL and Gil $S$ (2011) Emotion and time perception: effects of film-induced mood. Front. Integr. Neurosci. 5:33. doi: 10.3389/fnint.2011.00033

Copyright (c) 2011 Droit-Volet, Fayolle and Gil. This is an open-access article subject to a non-exclusive license between the authors and Frontiers Media $S A$, which permits use, distribution and reproduction in other forums, provided the original authors and source are credited and other Frontiers conditions are complied with. 